Retrospective Study

\title{
Peripheral Nerve Stimulation for the Treatment of Chronic Intractable Headaches: Long-term Efficacy and Safety Study
}

Pyung B. Lee, MD, PhD ${ }^{1}$, Christian Horazeck, MD², Francis S. Nahm, MD, PhD', and Billy K. Huh, MD, PhD ${ }^{3}$

\footnotetext{
From: 'Department of Anesthesiology and Pain Medicine, Seoul National University Bundang Hospital, Seongnam, South Korea; ${ }^{2}$ Department of Anesthesiology, Duke University Medical Center, Durham, NC, USA; ${ }^{3}$ Department of Pain Medicine, The University of Texas MD Anderson Cancer Center, Houston, TX, USA

Address Correspondence: Billy K. Huh, MD, PhD Professor University of Texas MD Anderson Cancer Center Department of Pain Medicine

Unit 409

Houston, TX 77030-4009, E-mail:

bkhuh@mdanderson.org

Disclaimer: There was no external funding in the preparation of this manuscript.

Conflict of interest: Dr. Billy

Huh is a consultant for St. Jude Medical, Plano, TX.

This work has been presented

at the annual meeting of

the American Society of

Anesthesiologists, Oct. 2013, in San Francisco, CA.

Manuscript received: 12-27-2014 Revised manuscript received: 03-17-2015

Accepted for publication: 03-20-2015

Free full manuscript: www.painphysicianjournal.com
}

Background: Despite the various modalities available for treating headaches, typical therapy does not provide adequate pain relief for some patients.

Objectives: This study explored the efficacy and safety of long-term peripheral nerve stimulation (PNS) for intractable chronic headaches.

Study Design: Retrospective study of refractory headache patients at academic pain center.

Methods: The medical records of all patients $(N=46)$ permanently implanted with PNS between January 2005 and January 2012 were reviewed retrospectively. Patient records and phone interviews were used to quantify the levels of pain intensity, headache days per month, and overall patient satisfaction with treatment. The correlation between headache duration and effectiveness of PNS was also assessed.

Results: Pain intensity on the 11-point Numeric Rating Scale decreased from $7.60 \pm 1.73$ before implantation to $3.78 \pm 2.41,3.32 \pm 2.67,3.42 \pm 2.74$, and $2.04 \pm 2.27$ at one, 6,12 , and $>$ $12(19-98)$ months after implantation, respectively $(P<.001)$. The mean number of headache days per month decreased by about 14 days from the base line. No correlation $(r=-.33)$ was found between the number of years the patients had suffered from headaches and the efficacy of treatment.

Limitations: Due to the limitation of a retrospective study, the data collected via chart reviews and phone interviews are susceptible to selection and information biases.

Conclusions: PNS is an effective modality in the long-term management of intractable chronic headaches. Despite long histories of chronic headaches, the majority of patients had significant reductions in pain scores and the number of headache days per month. The outcomes were not dependent on the number of years the patients had suffered from headaches before PNS treatment.

Key Words: Migraine headache, refractory headache, chronic daily headache, occipital neuralgia, cluster headache, hemicranias continua, refractory headache, occipital nerve stimulation, peripheral nerve stimulation

Pain Physician 2015; 18:505-516 eadaches are one of the most commonly reported neurological symptoms (1). Currently available data point to a worldwide headache prevalence of $47 \%$. The lifetime prevalence of generalized headaches across the globe has been estimated to be $>66 \%$. Approximately $18 \%$ of women and $6 \%$ of men in the United States experience migraines at any given time, while $4 \%$ of the American population is affected by daily chronic headaches (2). It has been estimated that migraine patients alone miss 
more than 157 million workdays each year (3). Thus, headache-associated health care costs and disabilities are a significant burden to both patients and society $(2,3)$.

Recurrent headaches can be treated with both rescue and preventive pharmacological therapy, but these do not always prove to be efficacious. For example, in an estimated $1 \%$ of all patients suffering from chronic migraines, pain cannot be adequately controlled by either conventional or alternative therapies (4). Reasons for treatment failure include inadequate analgesia, intolerable side effects, and the development of drug tolerance. Patients whose headaches are severe ( $>7$ on the 11-point Numeric Rating Scale [NRS]) and show no or only a temporary response to medication, nerve blocks, or biofeedback are categorized according to the International Classification of Headache Disorders (ICHD-2) as suffering from intractable chronic headaches (4-6). The World Health Organization rates the disability of persons affected by headaches as equivalent to the disability experienced by a person suffering from paraplegia or end-stage renal disease (3).

Recent publications have suggested peripheral nerve stimulation (PNS) as an effective therapeutic modality for controlling various forms of headaches (7). Historically, the use of PNS to treat headaches was introduced even before the gate control theory was published (8). In 1966, Shelden (9) implanted electrodes around the mandibular nerve to treat trigeminal neuralgia, producing temporary pain relief in his patients. A trial by Weiner and Reed (10) in 1999 led to the widespread use of occipital nerve stimulation to treat occipital neuralgia. They found good concordance between occipital nerve stimulation and pain relief in occipital neuralgia patients (10), although the headache disorder studied was subsequently reclassified as chronic migraine. Since then, other studies have extended the use of PNS treatment from occipital neuralgia to other headaches, such as migraines, cluster headaches, and hemicranias continua (11-13), with the stimulation of peripheral nerves being potential therapeutic targets $(14,15)$.

In their randomized multicenter prospective controlled study focused on occipital nerve stimulation for the treatment of intractable chronic migraine headache (ONSTIM), Saper et al (16) concluded that the results offered promise for further treatment approaches using this modality, although reliable conclusions regarding efficacy for greater than 3 months could not be established. Following these encouraging results, a large randomized prospective study using occipital nerve stimulation (ONS) to treat chronic migraine was published in 2012 (17). In this study, investigators reported a significant reduction in pain $(>30 \%)$, the average number of headache days per month, and migrainerelated disability. However, the study failed to reach the primary objective of a $50 \%$ or greater reduction in mean daily pain scores as measured by the visual analog scale 12 weeks after implantation.

Despite such encouraging results, the use of PNS to treat intractable headaches is still under investigation and has yet to be approved by the Food and Drug Administration (FDA). Many of the studies published to date lacked a follow-up of $>6$ months, suffered from a high rate of complications, or failed to address types of headache other than chronic migraines. Therefore, in this retrospective study, we evaluated the long-term efficacy and safety of PNS for chronic migraines and cluster headaches. We also analyzed the correlation between the duration of headache and response to PNS to help identify patients who can most benefit from this treatment.

\section{Methods}

\section{Patients}

The study was approved by the Institutional Review Board of the Duke University School of Medicine. The selection criteria for inclusion in this study were set as patients with a diagnosis of primary headache who had a PNS device implanted between January 2005 and January 2012. The patients' headache disorders were classified according to the criteria of the International Classification of Headache Disorders (second edition) (5).

The electronic medical record (EMR) for each patient was reviewed to document diagnosis, past interventional procedures (i.e., nerve blocks and/or radiofrequency ablation) with their efficacies, medication history, date of the PNS device implantation, implantation follow-up appointment remarks, and demographic data. The patients were contacted directly via phone for a brief, single interview regarding their headache disorder, previous procedures, and PNS use. All interviews were conducted by one physician. The reason for the interview was explained to patients, as well as the goals of the study, and patients were then asked to give verbal consent to participate in this interview. The interviewer read questions from a standard patient questionnaire (see Appendix) as written and 
without further comment. Questions could be repeated and medical terms explained in more colloquial terms, if requested by the patient. Attempts were made to minimize bias during the interview by sticking closely to the pre-written questionnaire and avoiding questions phrased differently from one respondent to the next. An interviewer was also asked to be systematic and consistent in interacting with each respondent and refrain from giving personal opinions. All answers were recorded in writing only. Interviews lasted between 5 and 10 minutes each. Up to 10 attempts were made to contact each patient during different times of the day. If contact could not be established or no valid phone number was available, the patient was considered to be unreachable. For patients who were not reachable, all data points used in this study were acquired from their EMRs.

\section{Efficacy}

Multiple measures were used to evaluate the efficacy of PNS against intractable headache disorders. Pain intensity was measured on the 11-point NRS, with 0 corresponding to "no pain" and 10 corresponding to "the worst pain imaginable." Pain intensity scores were acquired from EMRs for clinic visits pre-implantation and at one, 6 , and 12 months post-implantation. A final NRS score was acquired at the time of the interview. For patients whose PNS device was explanted, NRS scores were not acquired post-explantation.

As a second measure of efficacy, during the interview patients were asked to indicate the number of headache days per month before and after the implantation. If available, the number of headache days per month pre-implantation was obtained from the EMR to reduce recall bias. The number of headache days per month post-implantation (the most recent month) was obtained during the interview.

As a third measure of efficacy, patients were asked during the interview about any decreases in the number or dosage of pain medications after the implant. From the EMR and, if possible, the interview, the total number of medications, which included acute and preventive prescription medications (but not vitamins or supplements) intended specifically for the direct treatment of headache pain or associated signs and symptoms (e.g., nausea, sympathetic activity, or insomnia, and analgesics) as well as over-the-counter medications, was recorded and differentiated.

As a fourth measure of efficacy, patient satisfaction with the implantation of the PNS device and the associated outcomes was recorded. Satisfaction was measured on multiple levels. First, patients were asked whether or not they were satisfied with their PNS unit and the procedure as a whole. Patients were then asked to score their satisfaction on an 11-point scale, with 0 being "not satisfied at all" and 10 being defined as "complete satisfaction." To analyze patient satisfaction further, patients were asked whether they would be willing to undergo the procedure again in the future, considering their current experience, their experiences during the post-implantation period, and their perception of the procedure's efficacy. Patients were also asked whether they would recommend this procedure to a friend or family member if the procedure was offered to that person by a physician to treat chronic, intractable headaches. For patients whose PNS devices were removed before the time of the interview, the questions were adapted to focus on the period preceding explantation.

\section{Relationship between Headache Duration and Efficacy of PNS}

To measure the correlation between headache duration and PNS treatment efficacy, patients were divided into 2 groups: those with a history of $\leq 5$ years of daily headaches and those with a history of $>5$ years of daily headaches. The 5-year criterion was based on the findings of a previous study on spinal cord stimulation (18). An additional analysis examined previous successful interventional procedures, mainly peripheral nerve blocks, as prognostic factors for successful PNS implantation.

\section{Safety}

Multiple measures were used to evaluate the longterm safety of PNS for the treatment of intractable headache disorders. Each patient's EMR was screened for all post-implantation visit notes to identify any adverse effects, such as nausea, dizziness, lead migration, swelling, infection, or paresthesia. EMRs were further screened for any emergency room visits potentially related to post-implantation complications. During the interviews, patients were asked to report any postimplantation complications, physician visits, and infections. Patients were specifically asked about postsurgical infections of the wound site or other, potentially associated, tissue infections. Lastly, for those patients whose PNS device had been explanted, the reason for the removal was documented. 


\section{Statistics}

Statistical analyses were performed using JMP Pro 10.0 software (SAS Institute, Cary, North Carolina). Wilcoxon signed-rank tests were used on matched pairs to compare pre-implantation NRS scores, NRS scores at one, 6, and 12 months post-implantation, and NRS scores at the time of interview. The differences between the number of headache days per month preand post-implantation were assessed by a matched pair Wilcoxon signed-rank test, as normal distribution could not be inferred. Pre- and post-implantation NRS scores and the number of headache days per month were then analyzed by a matched pair Wilcoxon signedrank test in relation to patient gender. NRS scores and headache days per month were grouped by headache type, stimulator type, and stimulator laterality. KruskalWallis tests were used to compare groups for significant differences. A correlational analysis was performed to assess the relationship between the patients' preimplantation headache history and treatment response to the previous interventional procedures (e.g., nerve block or radiofrequency ablation). Patients were divided into groups based on their history of $>5$ or $\leq 5$ years of intractable headaches; between-group differences in NRS scores and in the number of headache days per month were assessed by a Wilcoxon rank sum test. All statistical tests were performed with $P=0.05$.

\section{Results}

\section{Patients}

During the study's time period, 60 patients underwent a trial for the implantation of PNS for the relief of intractable chronic headache. Among them, 14 cases failed to meet the screening criteria, leaving 46 patients included in the study after the initial screening; 35 of the 46 patients were available for a telephone interview. No patient refused to participate in the phone interview. Data for the remaining 11 patients were collected from the EMRs as they were not available for phone interview. The types of headache disorders were as follows: 35 patients had chronic migraines and 11 patients had cluster headaches. The latter group reported persistent headaches even after $\mathrm{O} 2$ treatments. Sixteen patients had a $\leq 5$-year history of headaches, and 30 patients had a $>5$-year history of headaches. At the time of the interview, the mean time interval since implantation was 52 months, with a range of between 19 and 98 months. Table 1 shows all patient demographics and characteristics.

PNS devices were implanted in various sites according to the patient's pain location. No statistical differences in electrode location were found based on gender or age. Table 2 lists the electrode position in detail and Fig. $1 A, B, C, D, E$ shows representative images of each PNS electrode's location. In 40 of the patients, the PNS

Table 1. Patient demographics and characteristics.

\begin{tabular}{|l|c|c|c|}
\hline & $\begin{array}{c}\text { Chronic Migraine } \\
(\mathbf{N}=\mathbf{3 5})\end{array}$ & Cluster Headache (N = 11) & $\begin{array}{c}\text { Total } \\
(\mathbf{N}=\mathbf{4 6})\end{array}$ \\
\hline Age (years) & $50.2 \pm 14.1$ & $46.5 \pm 11.1$ & $49.3 \pm 13.5$ \\
\hline Gender (M:F) & $12: 23$ & $5: 6$ & $17: 29$ \\
\hline Severity of pain (NRS) & $7.2 \pm 1.7$ & $8.8 \pm 1.4$ & $7.60 \pm 1.7$ \\
\hline Duration of headache (years) & $10.8 \pm 10.2$ & $12.3 \pm 10.1$ & $28 \pm 4.1$ \\
\hline Headache days per month & $28.0 \pm 4.6$ & $28.1 \pm 4.0$ & $3.9 \pm 1.5$ \\
\hline No. of medications & $4.0 \pm 1.9$ & $3.9 \pm 1.4$ & 10.1 \\
\hline
\end{tabular}

All data are represented in mean \pm standard deviation format, and reflect the baseline, i.e. the pre-PNS implantation period.

Table 2. PNS electrode location.

\begin{tabular}{|l|c|c|c||}
\hline Location & $\begin{array}{c}\text { Chronic Migraine } \\
(\mathbf{N}=\mathbf{3 5})\end{array}$ & $\begin{array}{c}\text { Cluster Headache } \\
(\mathbf{N}=\mathbf{1 1})\end{array}$ & $\begin{array}{c}\text { Total } \\
(\mathbf{N}=\mathbf{4 6})\end{array}$ \\
\hline ON & 29 & & 29 \\
\hline SON & 4 & 6 & 10 \\
\hline Combined ON+SON & 2 & 2 & 4 \\
\hline ATN & & 2 & 2 \\
\hline Combined SON+ATN & & 1 & 1 \\
\hline
\end{tabular}

ON: Occipital nerve, SON: Supraorbital nerve, ATN: Auriculotemporal nerve 


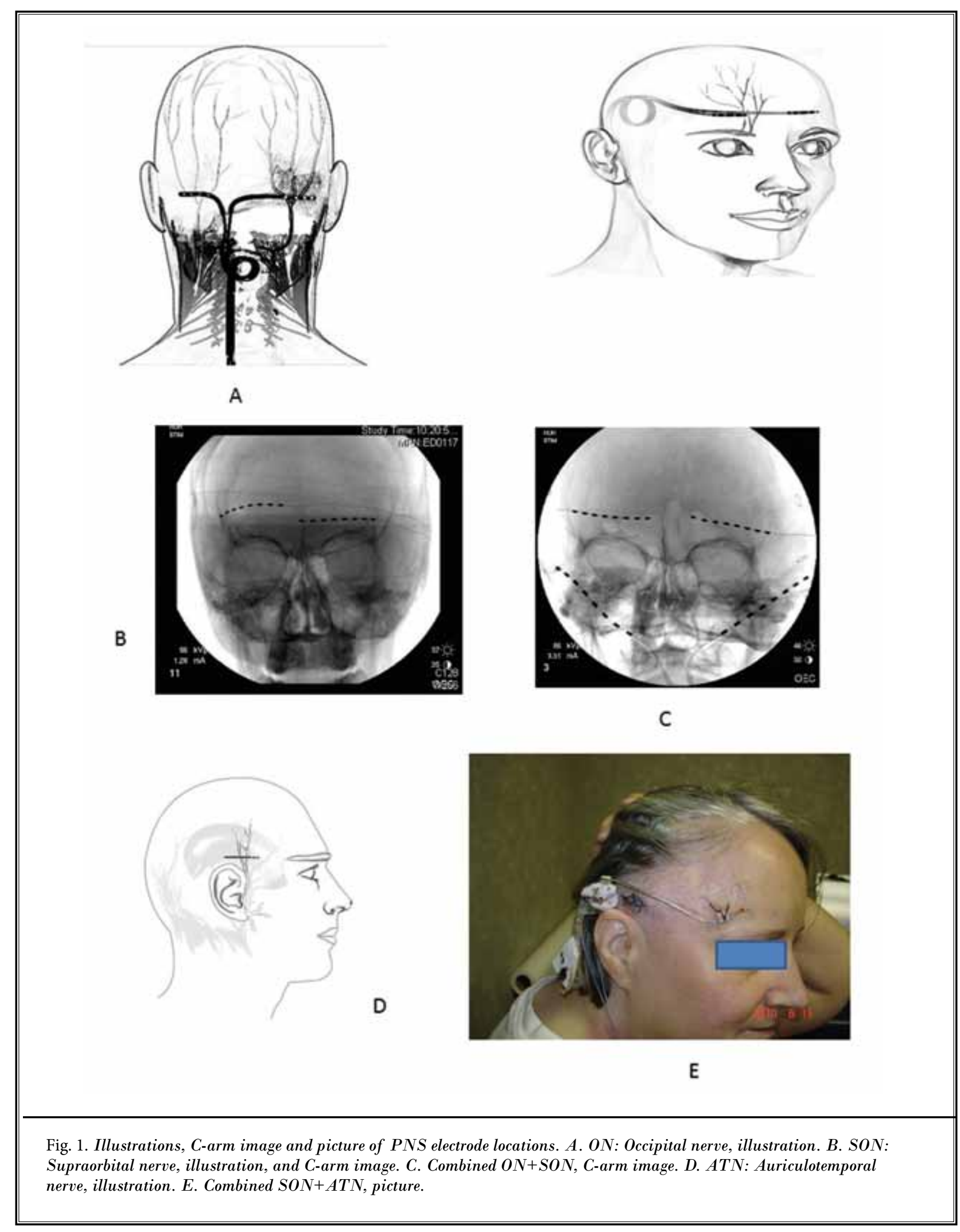


device remained implanted for more than 12 months; in the other 6 patients, the device was explanted within the first year.

\section{Efficacy}

Mean headache severity as measured by NRS scores decreased from $7.60 \pm 1.73$ to $3.78 \pm 2.41$ at one month post-implantation, $3.32 \pm 2.67$ at 6 months post-implantation, and $3.42 \pm 2.74$ at 12 months post-implantation ( $P<.001$, for all 3 comparisons; Fig. 2 shows the distribution and median NRS scores). The mean NRS score at the time of the interview (> 12 months post-implantation) was $2.04 \pm 2.27(P<.001)$. The mean NRS score differences between pre-implantation headache severities and headache severities at one, 6 , and 12 months postimplantation were 3.76, 4.29, and $4.15(P<.001$, for all 3 comparisons), respectively; the mean difference between the NRS scores obtained during the interview and the pre-implantation NRS scores was $5.56(P<.001$; Fig. 3). Men did not differ significantly from women in pre- or post-implantation scores. No significant differences were found among groups of NRS scores assigned by headache type, PNS electrode location, and laterality of PNS.

NRS scores obtained during the interviews were analyzed according to whether the interview took place $1-2,2-3,3-4,4-5,5-6$, or $>6$ years postimplantation; mean NRS scores were $2.05 \pm 2.27,2.12$ $\pm 2.45,2.07 \pm 2.23,2.18 \pm 2.44,1.89 \pm 1.96$, and $1.25 \pm$ 2.50 , respectively, and no statistically significant differences were found.

The mean number of headache days per month decreased from $28 \pm 4.1$ pre-implantation to $14.6 \pm 11.6$ post-implantation $(P<.001)$. The distribution of headache days prior to implantation followed a negatively skewed distribution, with a mode of the curve at 30 days/month, whereas post-implantation the distribution showed a positive skew with a mode of the curve at 5 days/month (Fig. 4). In addition, no significant differences emerged between men and women or among groups assigned by headache type, PNS electrode location, and laterality of PNS.

Before implantation, patients took an average of 4 different over-the-counter or prescribed medications for headaches or headache-associated symptoms. Of the 35 patients interviewed, $25(71 \%)$ were able to decrease the number and/or amount of medications used, although due to the difficulty in obtaining a detailed medication history for all patients, the extent of this decrease could not be quantified.

Thirty-two (91\%) of the 35 interviewed patients were satisfied with the PNS treatment. On a scale of 0

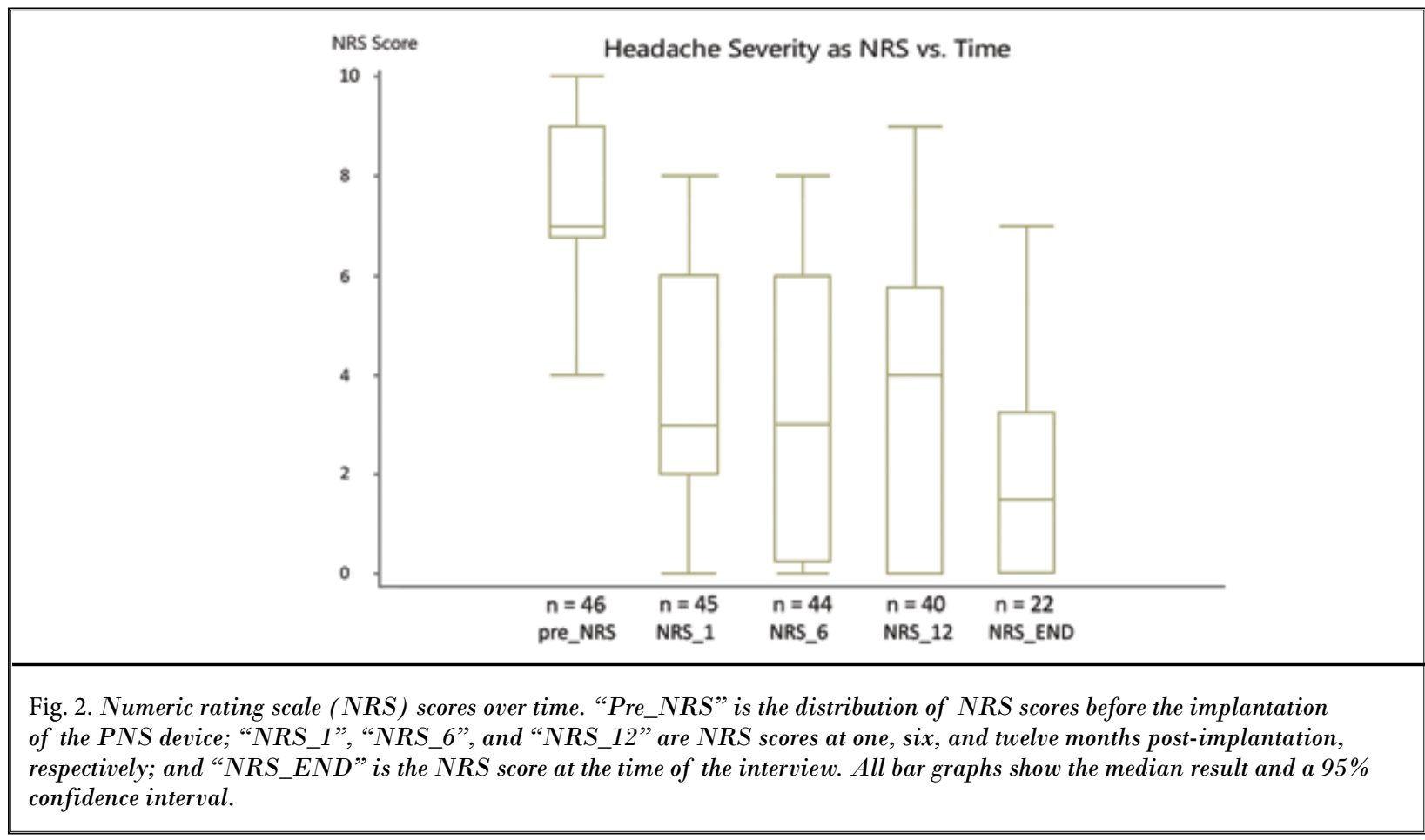


Peripheral Nerve Stimulation for the Treatment of Chronic Intractable Headaches

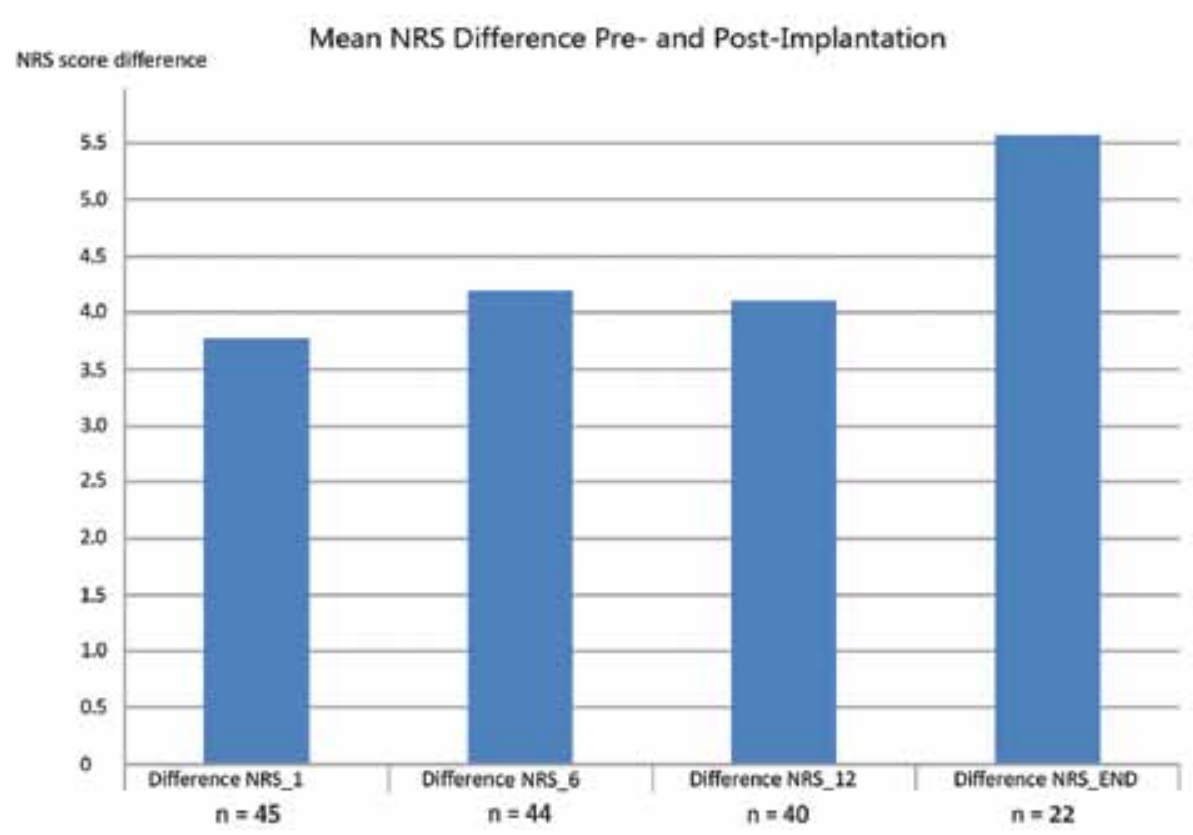

Fig. 3. Mean differences between the NRS scores before the implantation of the PNS device and the NRS scores at one, 6, and 12 months after implantation ("NRS_l", "NRS_6", and "NRS_12", respectively) and at the time of the interview ("NRS_ END").

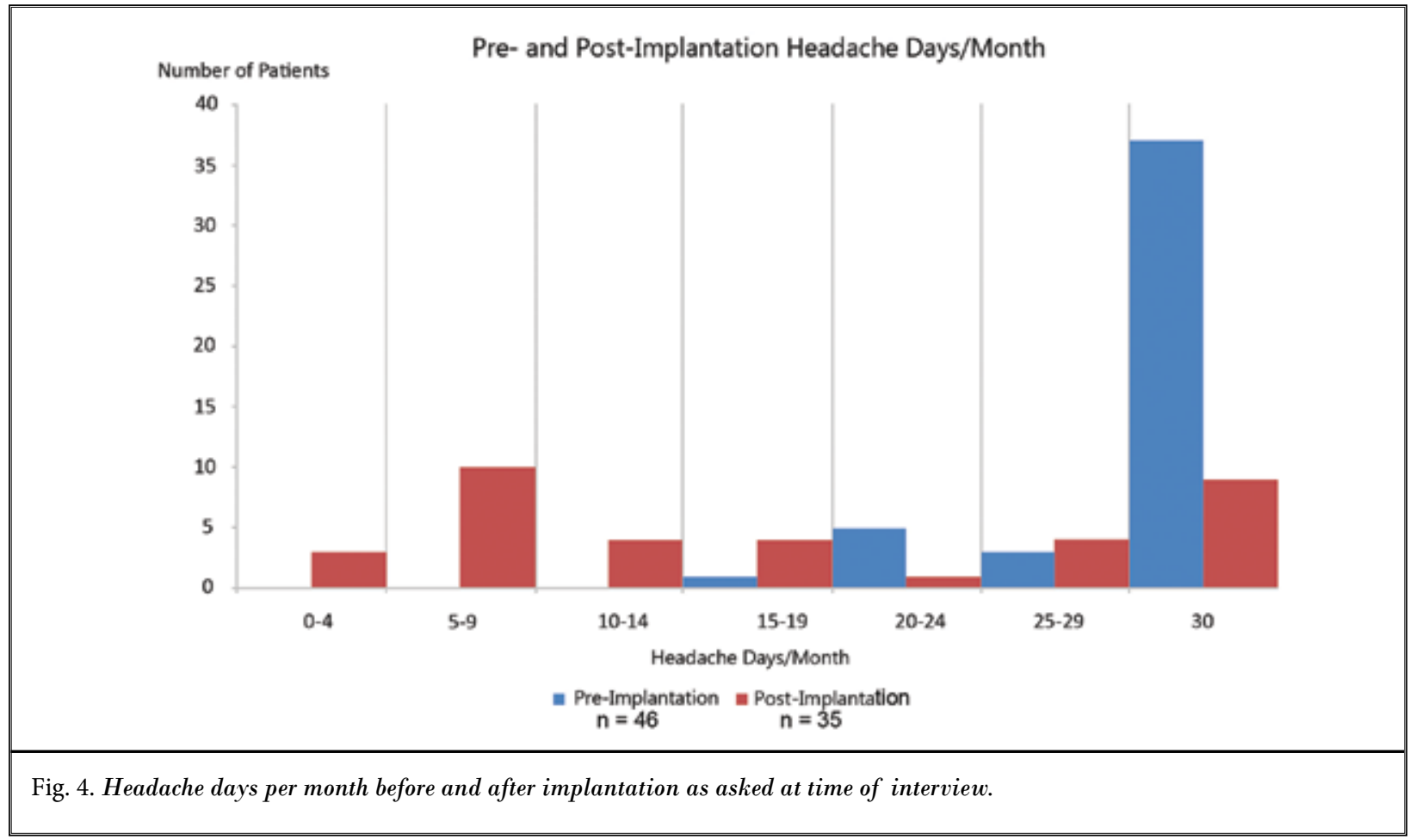




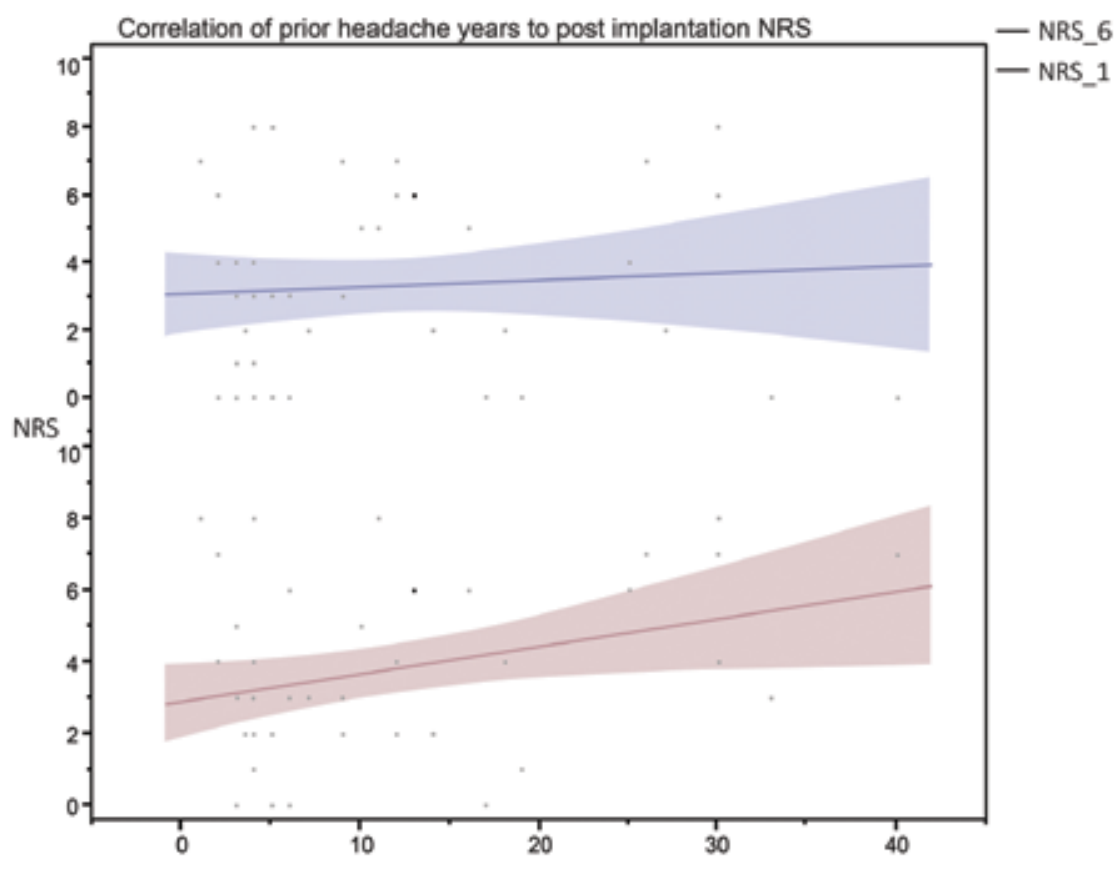

Fig. 5. Correlation between the patients' histories of headaches (numbers of years) and their NRS scores after the implantation of the PNS device. The 95\% confidence intervals are shaded. "NRS_1" and "NRS_6" mark the time points at one and six months post-implantation, respectively.

to 10 , with 10 being "completely satisfied," the mean score was $7.35 \pm 2.63$. Twenty-nine $(83 \%)$ of the patients said that they would recommend PNS treatment to a friend or family member with chronic, intractable headaches.

\section{Relationship between Headache Duration and Efficacy of PNS}

No correlation was found between the duration of intractable headaches and response to PNS treatment ( $r=-.33$ ) (Fig. 5). A reverse analysis of the relationship between positive PNS treatment responses and the number of years of intractable headaches found no correlation either. Patients with a > 5-year history of headaches did not differ significantly in NRS scores or headache days per month from patients with a $\leq$ 5 -year history of headaches. No correlation was found between previous successful interventional procedures, such as peripheral nerve blocks and responses to PNS treatment $(r=.14)$.

\section{Safety}

Surgical complications related to implantation were reported by 10 patients. Five of the 10 patients reported soreness in the implantation site; 2 patients reported migratory headaches; and one patient each reported pruritus, neck tightness, and paresthesia. With the exception of neck tightness, the side effects resolved in one month or sooner. A total of 15 longterm complications were reported: 2 patients had lead migration requiring revision; in one patient, the hardware failed; 3 patients reported worsening headaches after implantation; and 6 patients had surgical wound infections, 3 of which necessitated the removal of the PNS unit. Three patients reported unusual complications-namely, one device was destroyed in a physical altercation, one was damaged by the full body scanner at an airport security check, and one device was damaged during a neurosurgical procedure. All cases of infection were treated successfully with antibiotics or explantation, with no long-term sequela.

Sixteen patients had the PNS device removed. The reasons for removal were a decrease in efficacy over time $(N=7)$, lead migration or lead malfunction that could not be adequately corrected $(N=4)$, infection ( $N=$ $3)$, and complete resolution of headaches $(\mathrm{N}=2)$. There 
were no statistical differences between the group that underwent device explantation and the group in which devices remained implanted based on age, gender, or headache diagnosis. Table 3 summarizes adverse effects, complications, and explantation events.

\section{Discussion}

In our study, PNS treatment reduced mean headache severity by almost $50 \%$ within one month postimplantation. At 12 months post-implantation, headache severity decreased by at least $50 \%$ in 40 of the 46 patients $(87 \%)$. Beyond 12 months post-implantation, NRS scores continued to improve, and at the time of the interview (up to 98 months post-implantation) mean headache severity had decreased by $68 \%$ from pre-implantation levels. NRS scores reported during the interviews did not differ significantly with respect to time since implantation, indicating that the response to treatment was sustained.

The efficacy of PNS in treating intractable headaches is consistent with those of previous studies. Weiner and Reed reported an $80 \%$ pain score reduction in 13 patients with intractable occipital headache (10,
19). For occipital neuralgia, Slavin et al (20) reported that $70 \%$ of patients $(\mathrm{N}=10)$ experienced pain relief, while Melvin et al (21) reported that $91 \%$ of patients $(N=11)$ showed a reduction in medication requirements. In those studies, any improvement in the pain score was considered to be a positive response. In more recent studies, as in ours, a positive treatment response was defined as a $\geq 50 \%$ reduction in pain. This difference accounts for the higher treatment response rate in the earlier studies and makes the direct comparison of those studies with ours difficult.

In our study, $87 \%$ of the patients reported greater than $50 \%$ reduction in headache intensity with $50 \%$ reduction in number of headache days per month, from 28 days to $14(P=.001)$, after the implant. Similarly, a retrospective study $(\mathrm{N}=25)$ by Popeney and Alo (22) also showed $88 \%$ patients with greater than $50 \%$ reduction in headache intensity and $50 \%$ reduction in the number of headache days. The similarities between the 2 studies may be due to the fact that both are single center studies where only one implanter was involved in the procedure, thereby minimizing heterogeneity in implant technique.

Table 3. Side effects, complications, and reasons for explantation.

\begin{tabular}{|c|c|c|c|}
\hline & $\begin{array}{c}\text { Chronic Migraine } \\
(\mathbf{N}=\mathbf{3 5})\end{array}$ & $\begin{array}{c}\text { Cluster Headache } \\
\qquad(\mathrm{N}=11)\end{array}$ & $\begin{array}{c}\text { Total } \\
(\mathrm{N}=46)\end{array}$ \\
\hline Side effect & 7 & 3 & 10 \\
\hline Paresthesia & 1 & & 1 \\
\hline Neck tightness & & 1 & 1 \\
\hline Headache other site & 2 & & 2 \\
\hline Itching sensation & 1 & & 1 \\
\hline Implant site soreness & 3 & 2 & 5 \\
\hline Complication & 11 & 4 & 15 \\
\hline Infection & 5 & 1 & 6 \\
\hline Lead migration & 1 & 1 & 2 \\
\hline Headache aggravation & 2 & 1 & 3 \\
\hline Hardware fault & & 1 & 1 \\
\hline Miscellaneous & 3 & & 3 \\
\hline Explantation & 12 & 4 & 16 \\
\hline No further effect & 5 & 2 & 7 \\
\hline Infection & 2 & 1 & 3 \\
\hline Lead migration & 1 & 1 & 2 \\
\hline Lead malfunction & 2 & & 2 \\
\hline Resolution of headache & 2 & & 2 \\
\hline
\end{tabular}

Miscellaneous complications included one device broken through physical altercation, one device destroyed by magnetic fields during an airport security check, and one device damaged by a neurosurgical procedure. 
The number of analgesics and preventive medications, such as beta blockers, calcium channel blockers, antiepileptic medications, and muscle relaxants, used for headaches decreased in $71 \%$ of patients. There has been no direct cost assessment study between PNS and other therapy modalities, such as pharmacotherapy, nerve block, or physiotherapy, but data do suggest that PNS might be a cost-saving alternative when considering the overall cost of this condition. In the United States, the American Productivity Audit estimated the total economic burden of migraines to be $\$ 13$ billion annually (23). The American Migraine Prevalence and Prevention (AMPP) study estimated the annual cost of chronic migraines to be $\$ 7,750$ per person per year (24). In Ohio, the costs for Medicare in the reimbursement for the placement of a peripheral nerve stimulator, including all fees and the cost of the device in February 2011 , were estimated to be $\$ 26,578$, the majority of which is due to the cost of the device $(\$ 17,090)$. Jenkins and Tepper (25) calculated the cost effectiveness of the PNS procedure and concluded that this form of intervention seemed reasonable if the stimulation is utilized for patients in severe or disabling pain, is effective in those patients, and is continued for approximately 3 years. Although we could not directly calculate the cost benefit from the results, it could be inferred that PNS might provide cost relief for patients and payers during the management of intractable chronic headaches, along with its benefits in pain reduction.

Eighty-three percent of the patients (including some who underwent explantation and/or experienced complications) were willing to recommend PNS to friends and family members for the treatment of severe headaches. An average treatment satisfaction score of 7.63 on an 11-point scale was equally remarkable, as was the patients' overall satisfaction rate of $74.4 \%$. The reasons for dissatisfaction were, in decreasing order, the cost of the procedure, device malfunction, and unmet expectations.

The results of the significant reduction in both NRS scores and headache days per month after PNS implantation were unaffected by gender, type of headache, PNS target nerve, and use of unilateral/bilateral stimulators. This was to be expected, considering the comparability of results found in past publications that focused on different headache types, headache origins, and stimulator setups $(10,11,14,19-21)$.

As was reported in a previous study (26), the success of prior nerve blocks or other interventional procedures might not be a good predictor of the therapeutic effect of PNS, as we found no correlation between the reported success of those procedures and response to PNS $(r=.14)$. Patients who did not respond to occipital nerve blocks or radiofrequency ablation still showed a robust response to PNS.

Our study did not find a significant correlation between the number of years the patients suffered from headaches and the patients' responsiveness to PNS ( $P=$ -.33). The cutoff of 5 years was chosen on the basis of a previous study's finding that the classic cutoff margin used in the evaluation of spinal cord stimulation (<2-year history of chronic pain vs. $\geq 2$-year history of chronic pain) could not be used in the same way for PNS (18). Generally, studies on the efficacy of spinal cord stimulation found that the degree of pain relief was inversely related to the length of the period between the onset of pain and the time of implantation (27).

We attempted to differentiate minor post-implantation adverse effects that were linked directly to the surgical process, such as surgical site soreness, and/or resolved without further treatment within 14 days postoperatively following serious complications, such as infections. Most minor adverse effects were resolved by reprogramming the PNS device or with time. The most common PNS-associated complication is lead migration, reported to occur in $10 \%$ to $25 \%$ of patients treated with PNS $(20,28)$. In the largest multicenter randomized prospective study of PNS to date $(\mathrm{N}=177)$, Silberstein et al (17) reported that lead migration accounted for $18.7 \%$ of all adverse events. In our study, only 4 patients $(8 \%)$ had lead migration requiring revision, which provided successful results in 2 cases. This difference might be partially attributable to the difference in technique. For the patients in our study, a strain relief loop was applied to all implants with triple knots to secure the anchor to the lead and fascia. Moreover, the fact that all procedures were performed under the supervision of the same physician lowered the likelihood of inconsistencies in the application of the technique.

Kapural et al (29) reported that the lead migration rate for a midline incision was lower than the rate for a lateral incision. In addition, Shin et al (30) described an oblique cephalad trajectory for the midline incision to avoid sharp-angle positioning of the lead and minimize kinking or lead fracture. In the patients in our study, a similar midline incision technique was used, and all patients were fitted with a cervical collar during the first 7 days post-operatively to minimize neck movement. Sharan et al (31) analyzed adverse events in their ONSTM trial and reported that internal pulse generator 
Peripheral Nerve Stimulation for the Treatment of Chronic Intractable Headaches

Table 4. The efficacy and adverse events compared with other large studies $(N \geq 25)$.

\begin{tabular}{|l|c|c|c|c||}
\hline & $\begin{array}{c}\text { Number of } \\
\text { patients }\end{array}$ & $\begin{array}{c}\text { Efficacy(\% reduction is HA } \\
\text { intensity and HA days) }\end{array}$ & $\begin{array}{c}\text { Duration of follow up } \\
\text { (months) }\end{array}$ & Adverse events \\
\hline Saper et al $^{*}(2011)$ & 51 & $\begin{array}{c}>50 \% \text { reduction }(39 \%) \\
\text { Reduction in HA days }(24 \%)\end{array}$ & 3 & $\begin{array}{c}\text { Lead migration } 24 \% \\
\text { Infection } 18 \%\end{array}$ \\
\hline Silberstein et $\mathrm{l}^{*}(2012)$ & 105 & $\begin{array}{c}>50 \% \text { reduction }(17 \%) \\
\text { Reduction in HA days (28\%) }\end{array}$ & 3 & $\begin{array}{c}\text { Lead migration } 19 \% \\
\text { Infection } 4 \%\end{array}$ \\
\hline Popeney and Alo** (2003) & 25 & $\begin{array}{c}>50 \% \text { reduction }(88 \%) \\
\text { Reduction in HA days (50\%) }\end{array}$ & $9-36$ & $\begin{array}{c}\text { Lead migration } 36 \% \\
\text { Infection } 4 \%\end{array}$ \\
\hline Lee et al ${ }^{* *}$ (our study) & 46 & $\begin{array}{c}>50 \% \text { reduction }(87 \%) \\
\text { Reduction in HA days (50\%) }\end{array}$ & $19-98$ & $\begin{array}{c}\text { Lead migration } 8 \% \\
\text { Infection } 13 \%\end{array}$ \\
\hline
\end{tabular}

HA - headache

${ }^{*}$ Prospective study

${ }^{* *}$ Retrospective study

(IPG) implants closer to the lead location and implanters experience significantly reduced adverse events. These factors likely contributed to the low lead migration rate in our study.

Six patients in our study reported infections (13\%), and in 3 of those patients the device was removed as a result. This finding is consistent with the incidence of PNS-associated infection reported in other studies: $2 \%$ to $18 \%(28,32)$. Another reason for explantation was the decrease in efficacy over time. Generally, the revision rate for PNS appears to be higher than the revision rate for spinal cord stimulation $(20-21,28)$. Table 4 compares efficacy and adverse events of previous large studies $(\mathrm{N} \geq 25)$ with our study.

The weakness of our study is the heterogeneity in data collection. Although the use of phone interviews and EMRs allowed us to collect long-term follow-up data, phone interviews are susceptible to selection and information bias. The EMR often lacks important follow-up documentation. Attempts were made to minimize selection bias by limiting enrollment to patients who received PNS implantation from our clinic only.

\section{Conclusion}

In summary, our study found that PNS can offer long-term benefits for patients with intractable headaches and should be considered as a viable treatment option. The benefits of this procedure include reductions in headache intensity, the number of headache days per month, and the use of medications. The efficacy of PNS appears to be independent of the duration of headaches or the type of headache.

\section{References}

1. Goadsby PJ, Lipton RB, Ferrari MD. Migraine - Current understanding and treatment. $N$ Engl J Med 2002; 346:257-270.

2. Jensen R, Stovner LJ. Epidemiology and comorbidity of headache. Lancet Neurol 2008; 7:354-361.

3. Hu, ZH, Markson, LE, Lipton, RB, Stewart, WF, Berger ML. Burden of migraine in the United States. Arch Intern Med 1999; 159:813-818.

4. Irimia P, Palma JA, Fernandez-Torron R, Martinez-Vila E. Refractory migraine in a headache clinic population. BMC Neurology 2011; 11:94.

5. Olesen J. The International Classification of Headache Disorders. 2nd edition.. Prepared by Headache Classification Subcommitee of the international
Headache Society 2004.

6. Goadsby PJ, Schoenen J, Ferrari MD, Silberstein SD, Dodick D. Towards a definition of intractable headache for use in clinical practice and trials. Cephalalgia 2006; 26:1168-1170.

7. Goadsy PJ, Bartsch T, Dodick DW. Occipital nerve stimulation for headache: Mechanism and efficacy. Headache 2008; 48:313-318.

8. Melzack R, Wall PD. Pain mechanisms: A new theory. Science 1965; 150:971-979.

9. Shelden $\mathrm{CH}$. Depolarization in the treatment of trigeminal neuralgia. Evaluation of compression and electrical methods; clinical concepts of neurophysiological mechanism. In: Pain. Little, Brown, Boston, 1966, pp 373-386.
10. Weiner RL, Reed KL. Peripheral neurostimulation for control of intractable occipital neuralgia. Neuromodulation 1999; 2:217-221.

11. Magis D, Gerardy PY, Remacle JM, Schoenen J. Sustained effectiveness of occipital nerve stimulation in drug resistant chronic cluster headache. Headache 2011; 51:1191-1201.

12. Reed KL, Black SB, Banta CJ, Will KR. Combined occipital and supraorbital neurostimulation for the treatment of chronic migraine headaches: Initial experience. Cephalgia 2010; 30:260-271.

13. Schwedt TJ, Dodick DW, Trentman TL, Zimmerman RS. Occipital nerve stimulation for chronic headache and hemicranias continua: Pain relief and persistence of autonomic features. Cephalal- 
gia 2006; 26:1025-1027.

14. Amin S, Buvanendran A, Park KS, Kroin JS, Moric M. Peripheral nerve stimulator for the treatment of supraorbital neuralgia: a retrospective case series. Cephalalgia 2008; 28:355-359.

15. Simopoulos T, Bajwa Z, Lantz G, Lee S, Burstein R. Implanted auriculotemporal nerve stimulator for the treatement of refractory chronic migraine. Headache 2010; 50:1064-1069.

16. Saper JR, Dodick DW, Silberstein SD, McCarville S, Sun M, Goadsby PJ. Occipital nerve stimulation for the treatment of intractable chronic migraine headache: ONSTIM feasibility study. Cephalalgia 2010; 31:271-285.

17. Silberstein S, Dodick D, Saper S, Huh B, Slavin KV, Sharan A, Reed K, Narouze S, Mogilner A, Goldstein J, Trentman T, Vaisma J, Ordia J, Weber P, Deer T, Levy R, Diaz RL, Washburn SN, Merkhail N. Safety and efficacy of peripheral nerve stimulation of the occipital nerves for the management of chronic migraine: results from a randomized, prospective, multicenter, double-blinded, controlled study. Cephalgia 2012; 32:1165-1179.

18. Kumar K, Hunter G, Demeria D. Spinal cord stimulation in treatment of chronic benign pain: Challenges in treatment planning and present illness, a 22-year experience. Neurosurgery 2006; 58:481-496.

19. Weiner RL. Peripheral nerve neurostimulation. Neurosurg Clin N Am 2003; 14:401-408.

20. Slavin KV, Colpan ME, Munawar N,
Wess C, Nersesyan H. Trigeminal and occipital peripheral nerve stimulation for craniofacial pain: a single institution experience and review of the literature. Neurosurg Focus 2006; 21: $\mathrm{E}_{5}$.

21. Melvin EA, Jr Jordan FR, Weiner RL, Primm D. Using peripheral stimulation to reduce the pain of $\mathrm{C}_{2}$ mediated occipital headaches: A preliminary report. Pain Physician 2007; 10:453-460.

22. Popeney CA, Alo KM. Peripheral neurostimulation for the treatment of chronic disabling transformed migraine. Headache 2003; 43:369-375

23. Stewart WF, Ricci JA, Chee E, Morganstein D. Lost productive work time costs from health conditions in the United States: results from the American Productivity Audit. J Occup and EnvironMed 2003; 45:1234-1246.

24. Munakata J, Hazard E, Serrano D, Klingman D, Rupnow MF, Tierce J, Lipton RB. Economic burden of transformed migraine: results from the American Migraine Prevalence and Prevention (AMPP) Study. Headache 2009; 49:495-508.

25. Jenkins B, Tepper SJ. Neuoromodulation for primary headache disorders: Part 2, review of central neurostimulators for primary headache, overall therapeutic efficacy, safety, cost, patient selection, and future research in headache neuromodulation. Headache 2011 ; 51:1408-1418.

26. Schwedt TJ, Dodick DW, Trentman TL, Zimmerman RS. Response to occipital nerve is not useful in predicting efficacy of occipital nerve stimulation. Cephalalgia 2007; 27:271-274.

27. Kumar K, Toth C, Nath RK, Lating P. Epidural spinal cord stimulation for treatment of chronic pain; some predictors of success. A fifteen years experience. Surg Neurol 1998; 50:110-121.

28. Falowski S,Wang D, Sabesan A, Sharan A. Occipital nerve stimulator systems: Review of complications and surgical techniques. Neuromodulation 2010; 13:121-125.

29. Kapural L, Mekhail N, Hayek SM, Stanton-Hicks M, Malak O. Occipital nerve electrical stimulation via the midline approach and subcutaneous surgical leads for treatment of severe occipital neuralgia: A pilot study. Anesth Analg 2005; 101:171-174.

30. Shin JH, Kim YC, Jang IK, Kim JH, Park SY, Lee SC. Occipital nerve stimulation in a patient with an intractable chronic headache - a case report. KJP 2011; 60:298-301.

31. Sharen A, Huh B, Narouze S, Trentman T, Mogilner A, Vaisman J, Ordia J, Deer T, Venkatesan L, Salvin K. Analysis of adverse events in the management of chronic migraine by peripheral nerve stimulation. Neuromodulation 2015; 18:305-312.

32. Burher $A H$, Barnett $A H$, Barnett $C F$, Obray JB, Mauck WD. Introduction of infection control measures to reduce infection associated with implantable pain therapy devices. Pain Pract 2007; 7:279-284. 